\title{
Evaluation of Cylindrical Asymmetric Surface Dielectric Barrier Discharge Actuators for Surface Decontamination and Mixing
}

\author{
Alvin D. Ngo ${ }^{1, *,+}{ }^{\mathbb{D}}$, Kedar Pai ${ }^{2,+}$, Christopher Timmons ${ }^{2, \dagger}$, Li Maria Ma ${ }^{3}$ and Jamey Jacob ${ }^{1, * \mathbb{D}}$ \\ 1 Mechanical and Aerospace Engineering, Oklahoma State University, Stillwater, OK 74078, USA \\ 2 Plasma Bionics LLC, Stillwater, OK 74074, USA; kedarpai@plasmabionics.com (K.P.); \\ christimmons@plasmabionics.com (C.T.) \\ 3 Institute for Biosecurity and Microbial Forensics National, Oklahoma State University, \\ Stillwater, OK 74078, USA; li.ma@okstate.edu \\ * Correspondence: alvin.ngo@okstate.edu (A.D.N.); jdjacob@okstate.edu (J.J.) \\ + These authors contributed equally to this work.
}

check for updates

Citation: Ngo, A.D.; Pai, K.; Timmons, C.; Ma, L.M.; Jacob, J. Evaluation of Cylindrical Asymmetric Surface Dielectric Barrier Discharge Actuators for Surface Decontamination and Mixing. Plasma 2021, 4, 755-763. https://doi.org/ 10.3390/plasma4040038

Academic Editors: Savino Longo, Katharina Stapelmann and Pietro Ranieri

Received: 20 August 2021

Accepted: 16 November 2021

Published: 25 November 2021

Publisher's Note: MDPI stays neutral with regard to jurisdictional claims in published maps and institutional affiliations.

Copyright: (c) 2021 by the authors. Licensee MDPI, Basel, Switzerland. This article is an open access article distributed under the terms and conditions of the Creative Commons Attribution (CC BY) license (https:// creativecommons.org/licenses/by/ $4.0 /)$.

\begin{abstract}
Surface dielectric barrier discharge (SDBD) was used to evaluate cylindrical plasma actuators for inactivation of Salmonella enterica. A cylindrical SDBD configuration was evaluated to determine if the inherent induced body force could be leveraged to impel plasma species, such as reactive oxygen and nitrogen species (RONS), as an apparatus to sterilize surfaces. The cylindrical structure is evaluated in this study to observe whether an increase in mixing is possible to efficiently distribute the plasma species, thereby improving bacterial inactivation efficiency. The increase in induced airflow of SDBD actuators with increased numbers of electrodes correlates with increased bacterial inactivation. These results suggest that improving the particle velocity, airflow mixing tendencies, and plasma volume for the same power inputs (same net power to the actuators) results in increased surface decontamination efficiency.
\end{abstract}

Keywords: surface dielectric barrier discharge; cold atmospheric plasma; cylindrical actuators; bacterial inactivation; flow control; particle image velocimetry

\section{Introduction}

Cold atmospheric plasma (CAP), or nonthermal plasma produced at atmospheric pressure, has demonstrated utility in both surface decontamination and airflow control within the past two decades [1-5]. Although CAP may be generated through several methods, surface dielectric barrier discharge (SDBD) is one of the simplest means. Asymmetric SDBD actuators generate plasma between two asymmetrically placed electrodes, where at least one electrode is covered by a dielectric material (embedded) [4,5]. The main advantages of SDBD over other plasma generation methods are the low power requirements, customizability (design and scale), no gas-flow requirement, and most importantly a localized airflow induction generated from a corona discharge.

An asymmetric design of SDBD imparts momentum into the gas adjacent to the exposed electrode as a result of the electric field coupling with the surrounding gas. The gas is entrained, and an effective "induced flow" is produced. In a gaseous medium including atmospheric air, CAP induces the generation of reactive oxygen and nitrogen species, charged particles, and ultraviolet light. These are attributed as the major players contributing to bacterial inactivation observed in previous studies. Bacterial cellular damage results from high oxidative stress imposed on treated cells as a result of the action of the reactive species as well as electrostatic interactions of charged particles across the bacterial cell wall [2,6,7]. Ultimately, the cells die due to the constant barrage of accumulating reactive species, while being unable to keep up with lipid, protein, and DNA repair [8,9]. The induced flow of SDBD actuators functions as an effective delivery mechanism for the plasma-generated species to reach the contaminated surfaces [10]. By optimizing the geometric configurations of the plasma actuators, the SDBD impels the plasma-produced species toward a 
surface in a relatively power-efficient manner when compared to volumetric DBD (VDBD). Although VDBD has been utilized for bacterial inactivation, it requires a relatively higher power input, an addition of gas flow, and is highly limited in effective treatment area. VDBD lacks the natural means of species delivery that SDBD possesses, the induced fluid body flow. The true potential of SDBD is realized through the manipulation and utilization of the induced flow.

Several preliminary studies were performed to observe the efficacy of SDBD in different applications [10-14]. Various SDBD configurations were tested to improve induced flow strength and bacterial inactivation, including changes to physical parameters of electrodes and variations in dielectric materials. Geometrical changes in general considerably affect the total plasma volume output for a specific power input. For example, an increase in inter-electrode gap distance (i.e., the lateral distance between the exposed and embedded electrode) has been observed to increase induced flow strength, thereby increasing the effective treatment distance, but pushes the plasma into the "streamer regime", consequently increasing energy waste through heat and light dissipation [12,15]. Additionally, arranging electrode pairs in opposite directions allows for plasma jet vectoring, opening up a certain level of control for reactive species distribution and vectoring toward a specific surface. Through ideal electrode separation and power input, the plasma may be optimized into the "uniform regime" producing increased plasma volume per unit area [15]. Thus, there are a plethora of methods to increase the efficacy of SDBD for bacterial inactivation, whether it is the distribution method or the optimization of plasma species generation.

In this study, a cylindrical configuration was observed to determine whether an increase in fluid mixing, consisting of redistribution and homogenization of plasma generated species in the bulk of the fluid (in this case air), was feasible and whether this mixing had any impact on the inactivation of bacteria. In addition to this, mixing characteristics in different configurations with varying numbers of electrodes was observed to determine whether a correlation exists between number of electrodes and mixing efficiency. Increasing the number of electrodes effectively increases the sources for plasma to form and thus momentum entrainment locations. Particle image velocimetry (PIV) was used to evaluate the degree to which the airflow in the cylinder-shaped actuators is entrained and redistributed by the induced flow due to the plasma, observed through characteristics such as vorticity. Comparisons were made between one, two, three, and six electrodes within similar-sized cylindrical-shaped actuators. All actuators were driven with the same amount of total power. By increasing the number of electrodes, the total power to each electrode was lower, but the combined power remained the same. Naturally, an increase in electrodes resulted in an increase in the plasma surface area but decreased plasma intensity per unit area. The efficacy of the actuators to decontaminate surfaces was evaluated by observing the average log reduction of bacterial cultures after plasma treatment.

\section{Materials and Methods}

\subsection{Actuator Material and Design}

The cylindrical actuators used in this experiment were created using thin Teflon sheets, viewfoil made from polyester, and copper tape for the electrodes. The viewfoil was critical to this experiment to allow laser light to penetrate into the cylinder. Half the cylinder was viewfoil, while the other half was Teflon to act as the dielectric for the surface discharge actuator. The actuators were relatively circular with a diameter of around $1 \mathrm{inch}$. The actuators were constructed in an asymmetric configuration to allow for stronger induced flow. Each actuator configuration is displayed at the start of each electrode configuration PIV analysis, respectively. In the PIV experiment and the biological experiment, ambient air was used as the gas medium.

The electronics powering the actuator consisted of a minimax70 transformer (Information Unlimited, Amherst, NH, USA) running at $7 \mathrm{kV}$ and $10 \mathrm{~mA}$ and a $4.8 \mathrm{~V} 2000 \mathrm{mAh}$ battery pack (Tenergy, Fremont, CA, USA), as seen in Figure 1. The transformer utilizes a 
five-stage multiplier and can easily generate $30 \mathrm{kV}$ at $0.5 \mathrm{~mA}$ with the output at $25-35 \mathrm{kHz}$. The power going into the actuator is high-voltage AC.

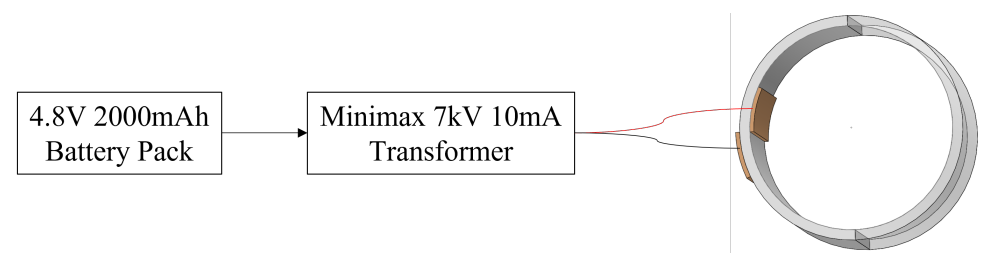

Figure 1. Flowchart of the electronics setup for the actuator.

\subsection{Particle Image Velocimetry}

Particle image velocimetry (PIV) is a commonly used analytical technique used to evaluate bulk fluid velocity profiles. In this experiment, the PIV setup utilized a Big Sky Laser Ultra Duel Nd:YAG laser that was connected to a pulse generator along with a high-speed Motion Pro X3 camera, as seen in Figure 2. A Taitech DG-100 timing control unit was used to control a Quantum Composers Plus 9518 pulse generator for laser/camera alignment. A Tektronix TDS 2014B oscilloscope was connected between the pulse generator and the lasers to monitor pulse delays. A laser optical setup that consisted of a cylindrical rod and a mixture of convex and concave lenses was used to obtain a $<2 \mathrm{~mm}$ laser sheet thickness. A ChauvetDJ Hurricane 700 fog generator was used to seed the air for PIV measurements. The time delay, $\mathrm{dt}$, used for this specific experiment was $500 \mu \mathrm{s}$.

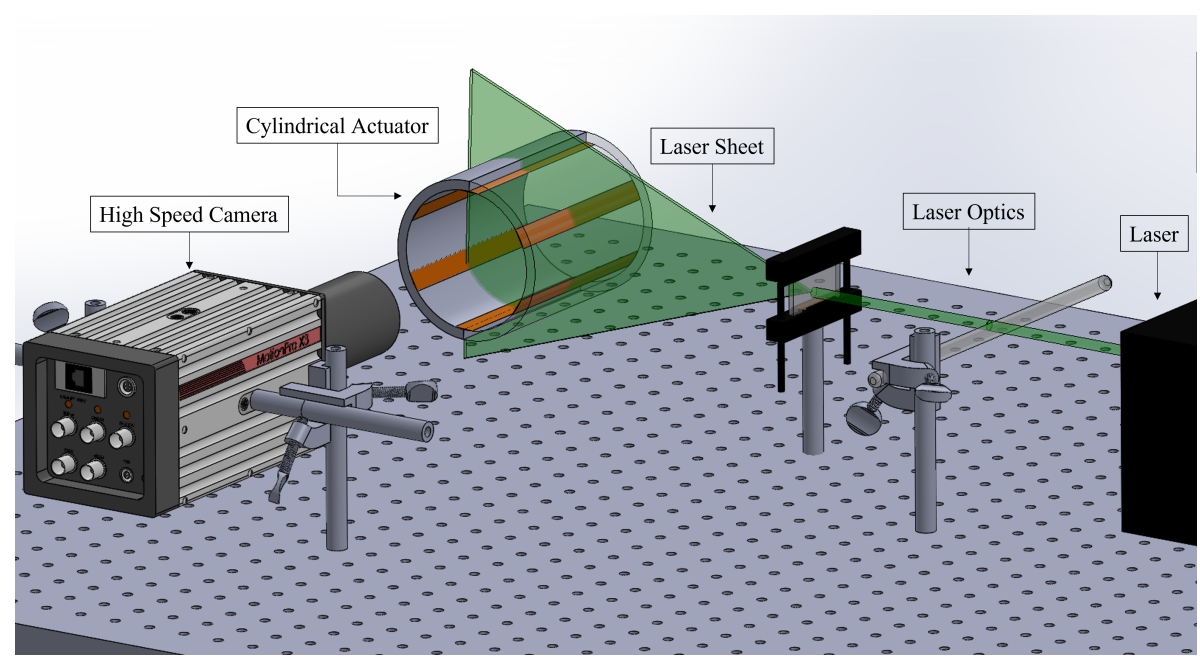

Figure 2. PIV setup schematic.

\subsection{Biological Experiment Setup}

For the bacterial inactivation section of this experiment, each cylinder configuration was used as a tube that enclosed a biological sample. The cylinders were rotated sideways and sat flat over the sample in a way to not touch the sample. A plastic cover was used to cover the open end of the cylinder. The entire setup was performed in a biosafety cabinet in a Petri dish. The setup is seen in Figure 3. 


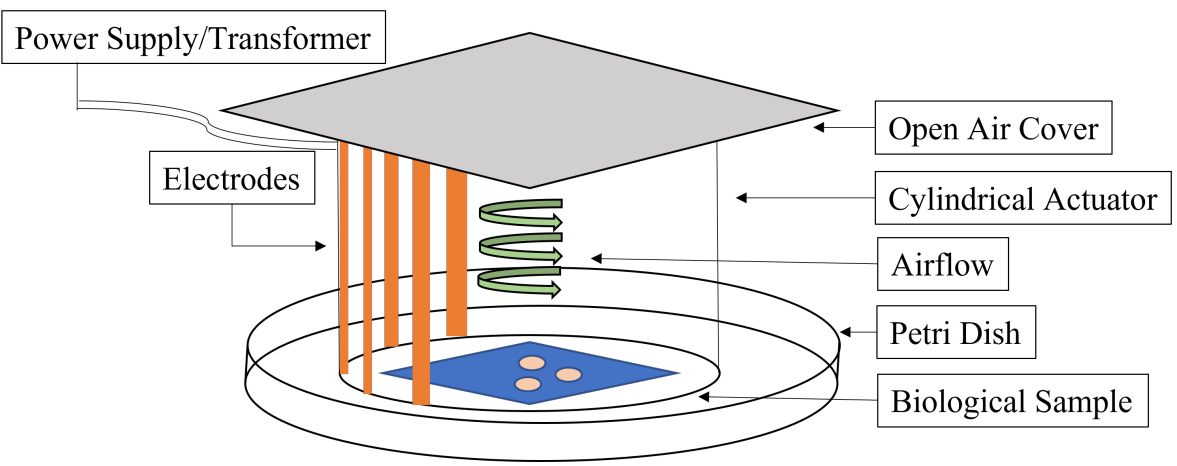

Figure 3. Schematic of the biological experimental setup.

\section{Results and Discussion}

\subsection{Particle Image Velocimetry}

Particle image velocimetry (PIV) analysis was used to evaluate the bulk fluid velocity profiles and mixing parameters within cylindrical SDBD plasma actuators with one, two, three, and six electrode arrays. The aim of PIV analysis was to determine the extent to which the actuators were capable of inducing airflow mixing. Since plasma-generated reactive species are known to have biocidal effects, an increase in airflow velocity and mixing capability would theoretically result in improved delivery of reactive species to contaminated surfaces, resulting in increased efficiency for surface decontamination applications. Although SDBD actuators can be constructed and arranged into any threedimensional structure, a cylindrical structure was used in this study to allow the optimal evaluation of airflow mixing capabilities since no edges or corners are present to influence the airflow dynamics. Additionally, the number of electrodes was varied from between one and six to evaluate the effects of increased plasma volume on the airflow velocity and mixing capabilities. The higher resolution in lower electrode numbers (one, two, and three electrodes) was to observe minute differences between each, while the larger electrode number of six was utilized to observe the upper limit of mixing. To compare the amount of mixing between the various electrode arrangements, several parameters were examined, including vorticity, streamlines, velocity magnitude, and root mean squared (RMS) velocity fluctuation.

For the one-electrode actuators, a strong single vortex was generated near the actuator that entrained only the flow close to the walls (Figure 4). Note that the actuator is positioned in the same location across all plots and their respective schematic. A highly concentrated vortex was clearly visible near the location of the actuator interface where the plasmainduced wall jet is generated (Figure $4 \mathrm{~b}$ ). As expected, this was also the location of maximum particle velocity (Figure $4 \mathrm{c}$ ) and velocity fluctuation (Figure $4 \mathrm{~d}$ ), indicating a higher degree of mixing.

When two-electrode actuators were used, the flow properties were more uniform within the entire cylindrical region (Figure 5). However, unlike the single small, strong vortex close to the electrode in the one-electrode arrangement (Figure 4c), two larger but comparatively weaker vortices were observed in the two-electrode arrangement (Figure 5c).

Accordingly, when three electrodes were used, three vortices were observed-two of which were considerably smaller than the third (Figure 6). The largest vortex at the center of the cylinder is presumed to influence the plasma-produced particles and redistribute them throughout the cross-section of the flow (Figure 6c). This characteristic results in a more uniform distribution of plasma-generated reactive species and more efficient surface decontamination potential.

The jump to six electrodes continued this trend with the most complete redistribution of particles in the flow (Figure 7). An increase in electrodes also comes with an increase in locations for the induced flow to originate from. Characteristically, more electrodes contribute to an increase in plasma surface area and volume, resulting in a higher concentration of reactive species in the flow. 
a)

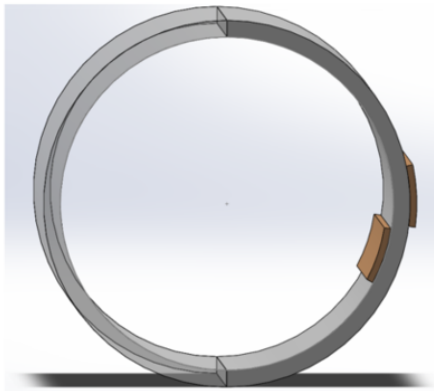

c)

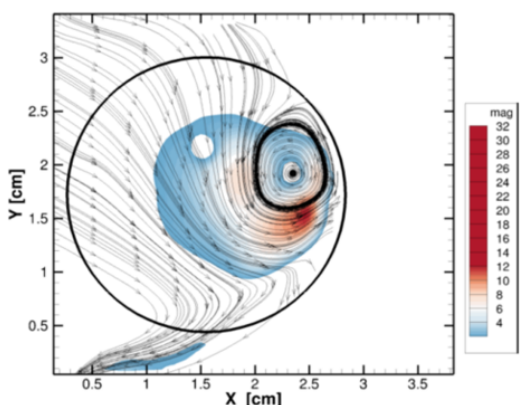

b)

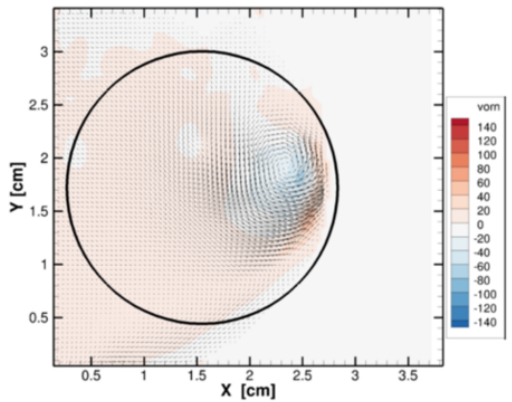

d)

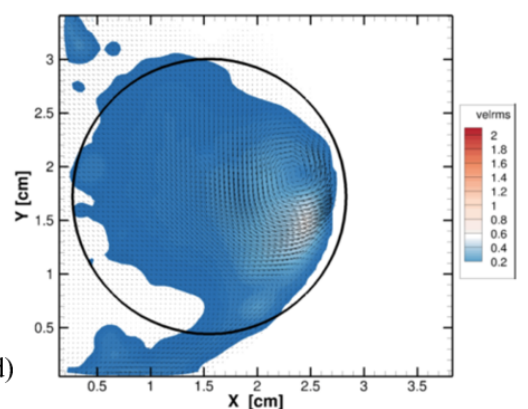

Figure 4. (a) Schematic of one electrode (1E) actuator (not to scale), (b) 1E vorticity plot, (c) $1 \mathrm{E}$ streamline and velocity magnitude plot, and (d) 1E RMS velocity plot.

a)

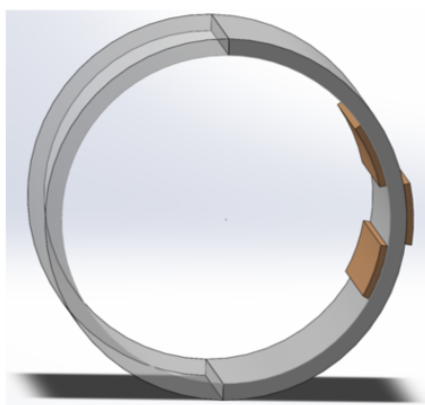

c)

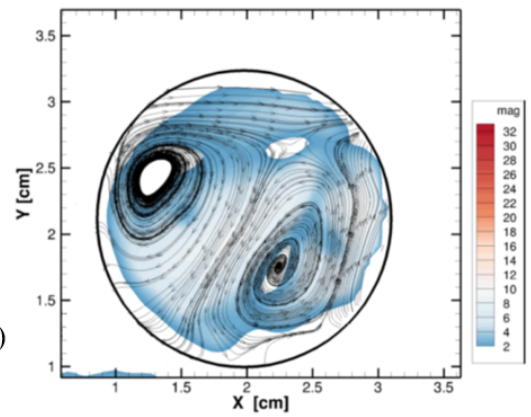

b)

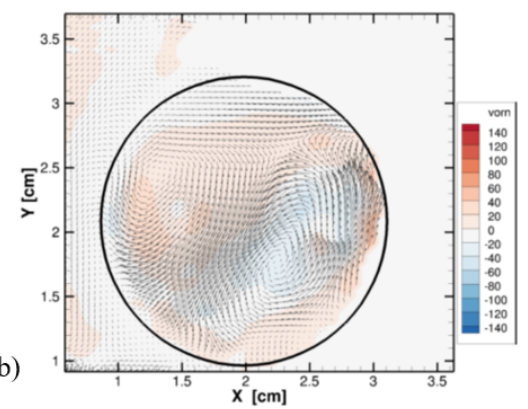

d)

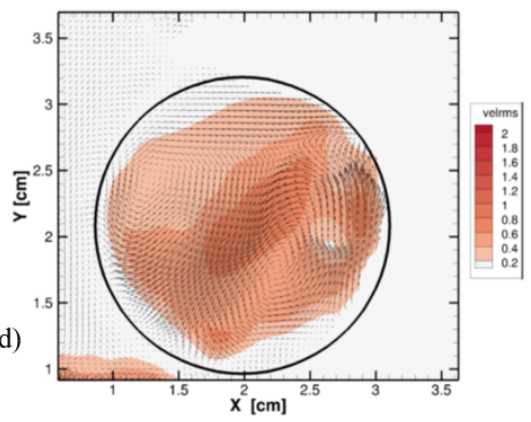

Figure 5. (a) Schematic of two electrode (2E) actuator (not to scale), (b) 2E vorticity plot, (c) $2 \mathrm{E}$ streamline and velocity magnitude plot, and (d) 2E RMS velocity plot.

These results suggest that increasing the number of electrodes and strategic positioning of the electrodes allows greater circulation and promotes better distribution of the induced airflow. Increasing the number of electrodes resulted in increased airflow uniformity and greater airflow mixing within the entire cylindrical region (Figures 4-7). This increased airflow uniformity may be attributed to the coupling of the flow of two adjacent oppositeacting actuators that result in a plasma jet directed perpendicularly to the surface. Increased mixing was primarily observed at the point of convergence of streamlines where the two vortices met (Figures $5 \mathrm{c}$ and $6 \mathrm{c}$ ). Thus, a higher number of vortices resulted in increased velocity variation and improved mixing tendencies (Figures $5 \mathrm{~d}$ and $6 \mathrm{~d}$ ). 
Further analysis of the flow field without a cylindrical enclosure revealed that the flow is perpendicular to the semicylindrical actuator surface and the vortices are a result of the obstruction formed by the remainder of the semicylindrical enclosure formed by the transparent shell. These results suggest that increasing the number of electrodes and strategic positioning of the electrodes allows for an increased circulation and optimal mixing of the flow.

a)

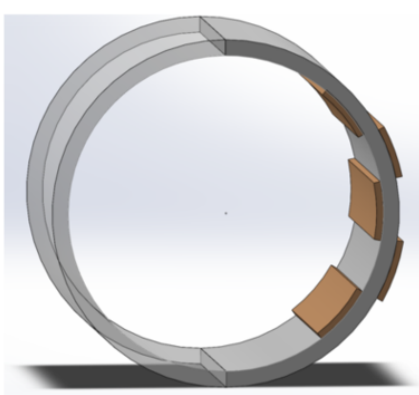

c)

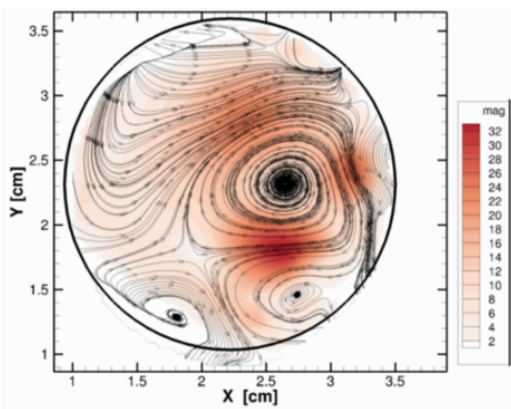

b)

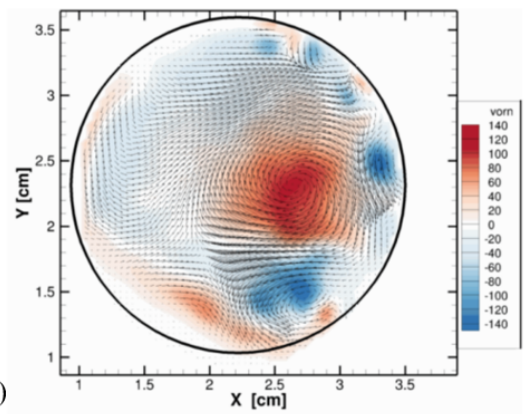

d)

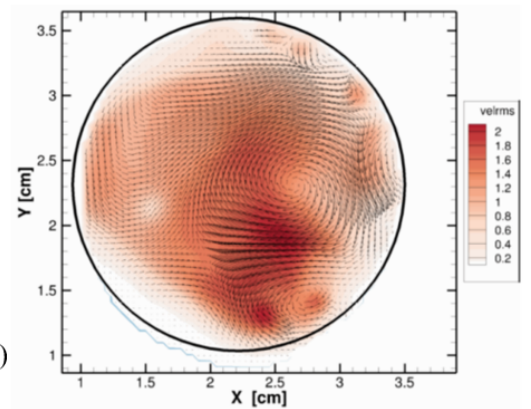

Figure 6. (a) Schematic of three electrode (3E) actuator (not to scale), (b) 3E vorticity plot, (c) 3E streamline and velocity magnitude plot, and (d) 3E RMS velocity plot.

a)

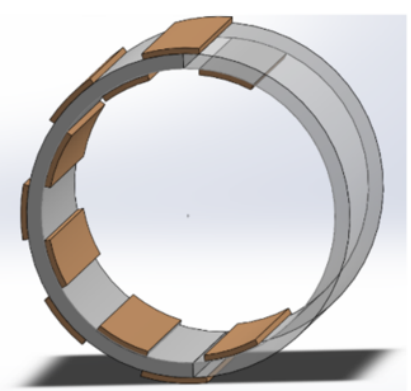

c)

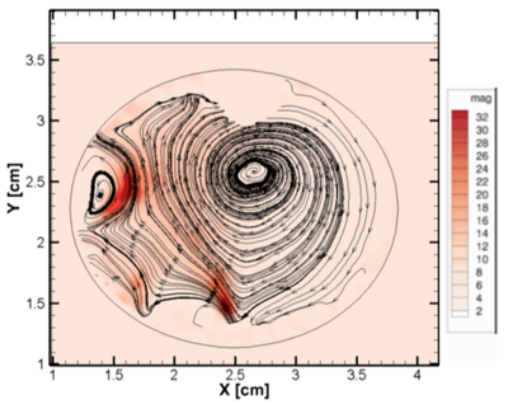

b)

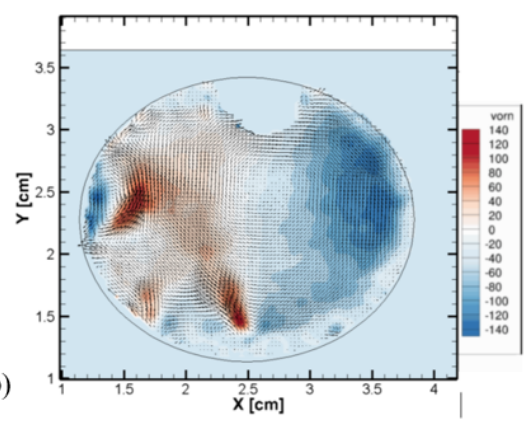

d)

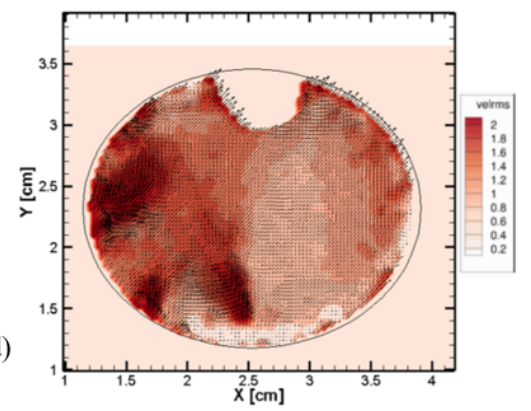

Figure 7. (a) Schematic of six electrode (6E) actuator (not to scale), (b) $6 \mathrm{E}$ vorticity plot, (c) $6 \mathrm{E}$ streamline and velocity magnitude plot, and (d) 6E RMS velocity plot.

\subsection{Bacterial Inactivation by Cylindrical SDBD Actuators}

All four SDBD actuator arrangements, utilizing one, two, three, and six electrodes, reduced Salmonella enterica populations on the inoculated coverslips after four minutes 
of treatment. Average log CFU mL-1 reductions of Salmonella cells were 1.30, 1.54, 1.81, and 2.28 for the one-, two-, three-, and six-electrode actuator arrangements, respectively (Figure 8). The average bacterial log reductions for the one-, three-, and six-electrode arrangements were significantly different from each other, while the log reduction from the two-electrode arrangement was only significantly different from the six-electrode arrangement $(p \leq 0.05)$. These data confirm that the increase in induced airflow of SDBD actuators with increased numbers of electrodes correlates with increased bacterial inactivation. These results suggest that by improved particle velocity, increased airflow mixing, and increased plasma volume results in increased surface decontamination efficiency. It should be noted that bacterial-inoculated coverslips were treated in line with the airflow and particle motion in this study. It is suspected that an increased bacterial reduction efficiency would have been observed if inoculated coverslips were placed perpendicular to the airflow and particle motion. A perpendicular orientation of contaminated substrate to plasma-induced airflow would allow a more direct bombardment of plasma-produced reactive species and would maximize the cell-damaging effects of these particles for surface decontamination applications.

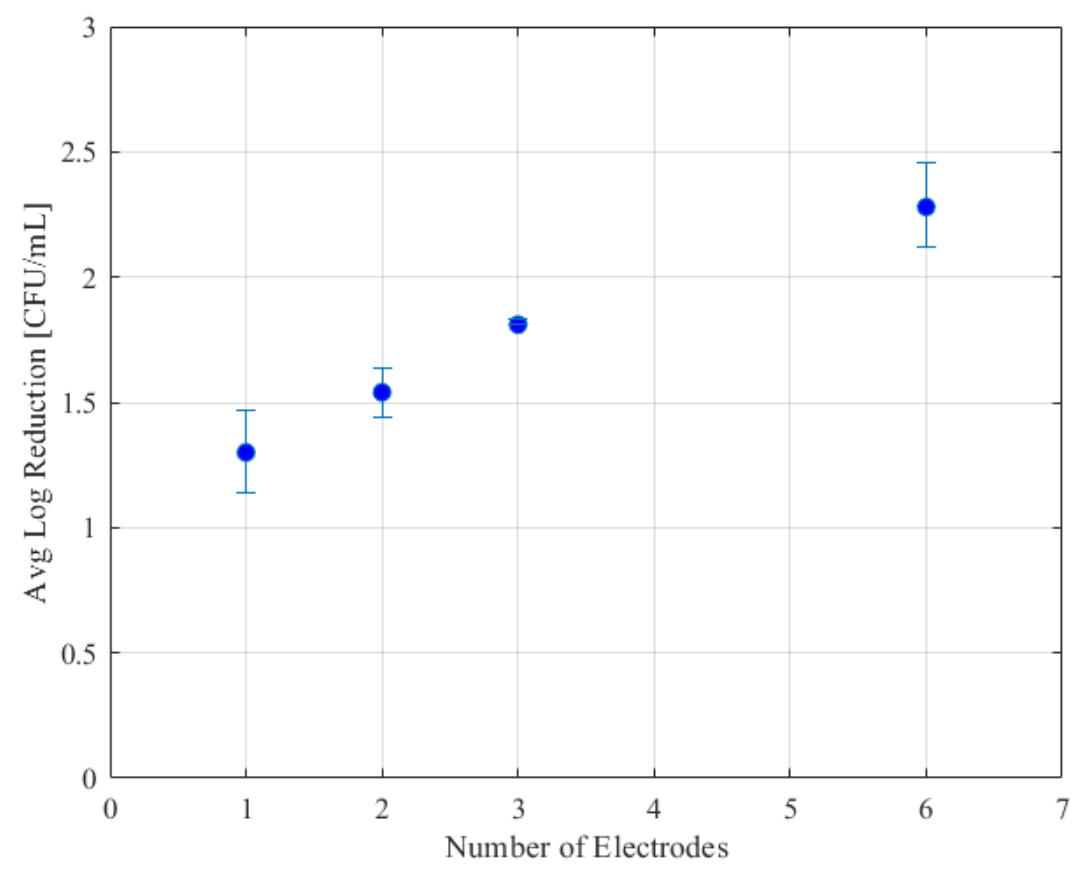

Figure 8. Average log reduction (CFU mL ${ }^{-1}$ ) of Salmonella cells inoculated onto glass coverslips placed within SDBD actuators with one, two, three, and six electrodes and treated for four minutes.

\subsection{Assessment of Bacterial Inactivation by Cylindrical SDBD Actuators}

A five-strain mixture of Salmonella enterica subspecies enterica (serovars Enteritidis, Typhimurium, Javiana, Seftenburg, and Poona) was used for inoculation of sterile glass coverslips, which were then placed in the center of cylindrical SDBD actuators with one, two, three, and six electrodes and treated for $4 \mathrm{~min}$. Bacterial strains were grown aerobically overnight with shaking ( $250 \mathrm{rpm}$ ) at $37^{\circ} \mathrm{C}$ in $5 \mathrm{~mL}$ tryptic soy broth (TSB, Difco, Sparks, $\mathrm{MD}, \mathrm{USA})$. The bacterial concentration of each overnight liquid culture was determined by serially diluting the culture in $0.1 \%(w / v)$ sterile peptone (Difco, Sparks, MD, USA) and plating in duplicate on tryptic soy agar (TSA, Difco, Sparks, MD, USA), incubated overnight at $37^{\circ} \mathrm{C}$.

To prepare the inocula, $1 \mathrm{~mL}$ of each liquid culture was centrifuged at $9000 \times g$ for $3 \mathrm{~min}$ and resuspended in $1 \mathrm{~mL}$ of $0.1 \%(w / v)$ sterile peptone before being combined with all other strains. In addition, $100 \mu \mathrm{L}$ of the bacterial suspension (approximately $107 \mathrm{CFU}$ ) was used as inoculum and spotted in 20-25 spots (between 105 and $106 \mathrm{CFU} /$ spot) onto single 
sterile $22 \times 22 \mathrm{~mm}$ glass coverslips and dried in a biosafety cabinet for $90 \mathrm{~min}$ prior to cold plasma treatment. Immediately after cold plasma treatment, treated and untreated control inoculated coverslips were washed by vortexing for $30 \mathrm{~s}$ in $10 \mathrm{~mL} 0.1 \%(w / v)$ sterile peptone in $50 \mathrm{~mL}$ conical tubes. The wash fluid was ten-fold serially diluted in $0.1 \%$ peptone, and $100 \mu \mathrm{L}$ of appropriate dilutions was plated in duplicate on TSA and incubated overnight at $37^{\circ} \mathrm{C}$.

Three biological repeats containing two replicates of each treatment were conducted. Log reductions due to plasma treatment were calculated by comparing the numbers of recovered cells from treated samples and untreated controls. Statistical difference was calculated between treated samples and untreated controls from plate counts by using analysis of variance (ANOVA) with SAS (Statistical Analysis System. Inst. Inc., Cary, NC, USA). A significant difference was defined at $p \leq 0.05$.

\section{Conclusions}

In summary, using a cylindrical SDBD actuator, a correlation between an increase in the number of electrodes and an increase in the degree of mixing was observed. The increase in electrodes provides more wall locations for airflow to be entrained. Velocity fluctuations increase as well, demonstrating a higher degree of particle redistribution within the airflow. The total number of vortices perceived within the cylindrical structure increased with more electrodes. The area between vortical structures caused the highest fluctuation in velocities, potentially indicating mixing. Additionally, the increase in electrodes yields an increase in total plasma surface area, which is observed to correlate with an increase in bacterial inactivation. More plasma species were generated by the higher plasma volume, and the bacterial inactivation efficiency of these species were enhanced due to the higher particle redistribution rate. A relative decrease in power per plasma surface area in the actuators with more electrodes was not observed to play a role in plasma-based decontamination but needs to be analyzed for changes in the type of dominant species produced when higher power regimes are used. Increased power only produced streamers and heat in this study, which may not be beneficial for actuator longevity.

A cartoon schematic is illustrated below to summarize expected vortices from certain cylindrical electrode configurations (Figure 9). In the case of an odd number of exposed electrodes, the imperfections in the electrodes have one side dominant, thus altering the positions of the expected vortices. In future studies, cylindrical structures could be further explored in cases of electrodes all the way around the cylinder with the jet vectors, all directed inward along with various combinations of laid along the wall to accelerate or interrupt the flow. In this study, by utilizing a cylindrical configuration and using an increased number of electrodes, a higher efficiency SDBD actuator in terms of degree of airflow mixing is possible for bacterial inactivation. Potential applications of these findings span multiple disciplines for surface decontamination of complex structures.

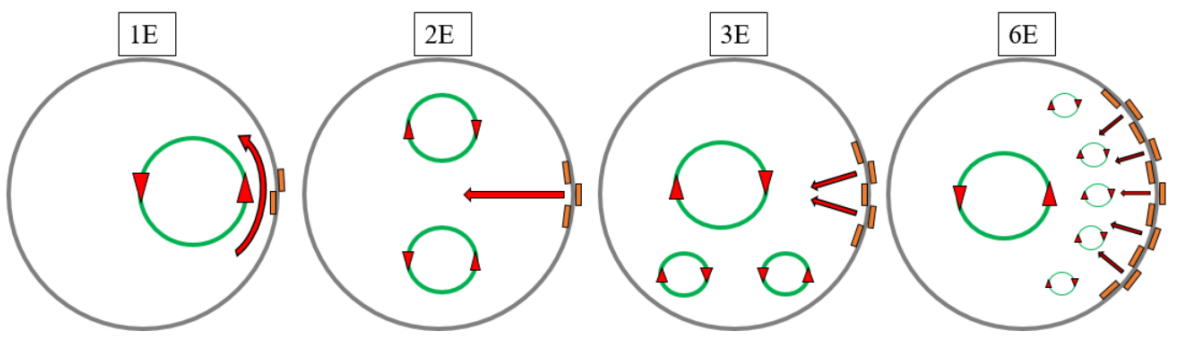

Figure 9. Cartoon schematic of expected vortices from one, two, three, and six exposed electrode cylindrical actuators.

Author Contributions: Conceptualization, K.P., C.T., L.M.M. and J.J.; metholodogy, formal analysis, validation, investigation, and data curation, A.D.N., K.P. and C.T.; resources and supervision, L.M.M. and J.J.; writing-original draft preparation, A.D.N., K.P. and C.T.; writing—review and editing, 
A.D.N., K.P., C.T., L.M.M. and J.J. All authors have read and agreed to the published version of the manuscript.

Funding: This research received no external funding.

Data Availability Statement: The data presented in this paper in the form of raw high-speed camera images used as inputs into PIV processing as well as the analyzed DaVis PIV velocity vectors are all available upon request from the authors.

Conflicts of Interest: The authors declare no conflict of interest. There are no conflicts of interest from the co-authors Kedar Pai and Christopher Timmons at Plasma Bionics with the work discussed in this paper. At the time of involvement with the project, they were not at Plasma Bionics LLC yet. Plasma Bionics LLC had no role in the design of the study; in the collection, analyses, or interpretation of data; in the writing of the manuscript, or in the decision to publish the results.

\begin{tabular}{|c|c|}
\hline \multicolumn{2}{|c|}{ Abbreviations } \\
\hline \multicolumn{2}{|c|}{ The following abbreviations are used in this manus } \\
\hline \\
\hline CAP & Cold Atmospheric Plasma \\
\hline PIV & Particle Image Velocimetry \\
\hline \multirow{2}{*}{$\begin{array}{l}\text { PWM } \\
\text { RMS }\end{array}$} & Pulse Width Modulation \\
\hline & Root Mean Squared \\
\hline \multirow{2}{*}{$\begin{array}{l}\text { RONS } \\
\text { SAS }\end{array}$} & Reactive Oxygen and Nitrogen Species \\
\hline & Statistical Analysis System \\
\hline \multirow{2}{*}{$\begin{array}{l}\text { SDBD } \\
\text { VDBD }\end{array}$} & Surface Dielectric Barrier Discharge \\
\hline & Volumetric Dielectrıc Barrier Discharge \\
\hline
\end{tabular}

\section{References}

1. Laroussi, M. Nonthermal decontamination of biological media by atmospheric-pressure plasmas: Review, analysis, and prospects. IEEE Trans. Plasma Sci. 2002, 30, 1409-1415. [CrossRef]

2. Moisan, M.; Barbeau, J.; Moreau, S.; Pelletier, J.; Tabrizian, M.; Yahia, L. Low-temperature sterilization using gas plasmas: A review of the experiments and an analysis of the inactivation mechanisms. Int. J. Pharm. 2001, 226, 1-21. [CrossRef]

3. Kong, M.G.; Kroesen, G.; Morfill, G.; Nosenko, T.; Shimizu, T.; van Dijk, J.; Zimmermann, J.L. Plasma medicine: An introductory review. New J. Phys. 2009, 11. [CrossRef]

4. Moreau, E. Airflow control by non-thermal plasma actuators. J. Phys. D Appl. Phys. 2007, 40, 605-636. [CrossRef]

5. Corke, T.C.; Enloe, C.L.; Wilkinson, S.P. Dielectric Barrier Discharge Plasma Actuators for Flow Control. Annu. Rev. Fluid Mech. 2010, 42, 505-529. [CrossRef]

6. Laroussi, M.; Alexeff, I.; Kang, W.L. Biological Decontamination by Nonthermal. IEEE Trans. Plasma Sci. 2000, $28,184-188$. [CrossRef]

7. Laroussi, M.; Leipold, F. Evaluation of the roles of reactive species, heat, and UV radiation in the inactivation of bacterial cells by air plasmas at atmospheric pressure. Int. J. Mass Spectrom. 2004, 233, 81-86. [CrossRef]

8. Herrmann, H.W.; Henins, I.; Park, J.; Selwyn, G.S. Decontamination of chemical and biological warfare (CBW) agents using an atmospheric pressure plasma jet (APPJ). Phys. Plasmas 1999, 6, 2284-2289. [CrossRef]

9. Boudam, M.K.; Moisan, M.; Saoudi, B.; Popovici, C.; Gherardi, N.; Massines, F. Bacterial spore inactivation by atmosphericpressure plasmas in the presence or absence of UV photons as obtained with the same gas mixture. J. Phys. D Appl. Phys. 2006, 39, 3494-3507. [CrossRef]

10. Timmons, C.; Pai, K.; Jacob, J.; Zhang, G.; Ma, L.M. Inactivation of Salmonella enterica, Shiga toxin-producing Escherichia coli, and Listeria monocytogenes by a novel surface discharge cold plasma design. Food Control 2018, 84, 455-462. [CrossRef]

11. Pai, K.K.; Singarapu, K.; Jacob, J.D.; Madihally, S.V. Dose Dependent Selectivity and Response of Different Types of Mammalian Cells to Surface Dielectric Barrier Discharge (SDBD) Plasma. Plasma Process. Polym. 2015, 12, 666-677. [CrossRef]

12. Ngo, A.; Pai, K.K.; Jacob, J.D. Investigation of Scaling Effects Due to Varying Dielectric Materials in Asymmetric Surface Dielectric Barrier Discharge. Aerosp. Res. Cent. 2017. [CrossRef]

13. Pai, K.K. Asymmetric Surface Dielectric Barrier Discharge as a Novel Method for Biological Decontamination. Ph.D. Dissertation, Oklahoma State University, Stillwater, OK, USA, 2015.

14. Pai, K.K.; Jacob, J.D. Evaluation of Dielectric Barrier Discharge Configurationsfor Biological Decontamination. Aerosp. Res. Cent. 2013. [CrossRef]

15. Enloe, C.L.; McLaughlin, T.E.; VanDyken, R.D.; Kachner, K.D.; Jumper, E.J.; Corke, T.C. Mechanisms and Responses of a Single Dielectric Barrier Plasma Actuator: Plasma Morphology. AIAA J. 2004, 42, 589-594. [CrossRef] 